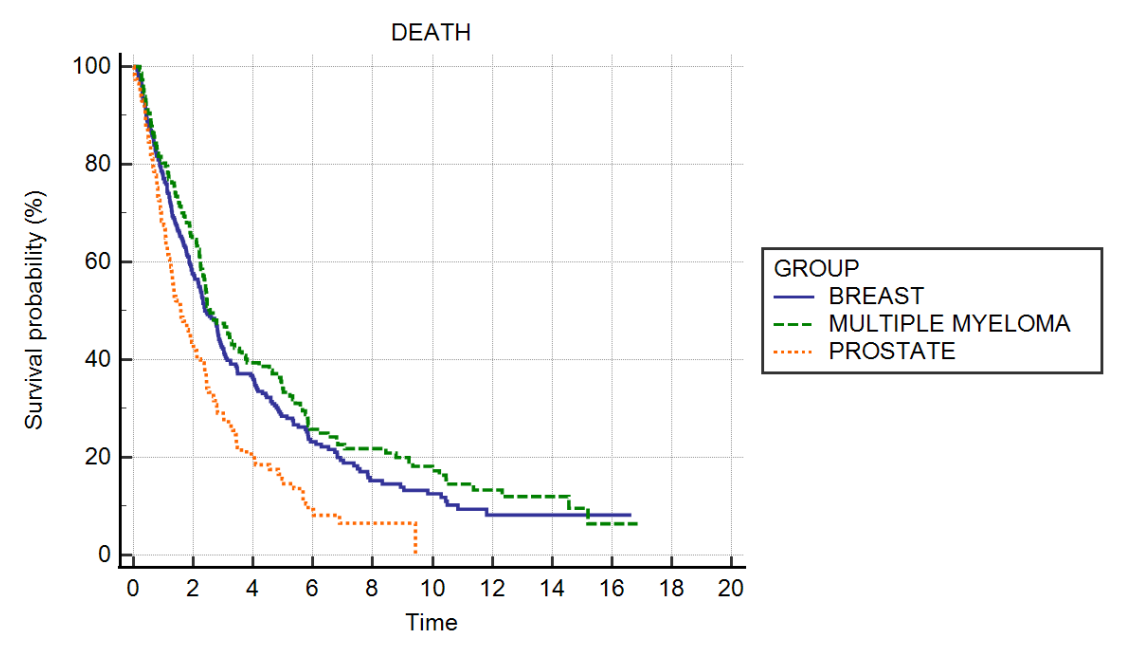

\title{
Survival of metastatic cancer and myeloma patients after diagnosis of Osteonecrosis of Jaws (ONJ). Potential impact on ONJ treatment strategies.
}

\author{
MARCO CABRAS ${ }^{1}$, Alessio Gambino ${ }^{1}$, DAVIDE CONROTTO ${ }^{1}$, Marco Garrone ${ }^{1}$, Antonella Fasciolo ${ }^{2}$, lorenzo \\ basano ${ }^{3}$, Marco Gilardetti ${ }^{4}$, claudia galassi ${ }^{4}$, Vittorio Fusco $^{2}$ \\ 1 University of Turin \\ 2 Azienda Ospedaliera SS. Antonio e Biagio e Cesare Arrigo \\ 3 Azienda Ospedaliera Ordine Mauriziano di Torino \\ 4 Centro di Riferimento per l'Epidemiologia e la Prevenzione Oncologica in Piemonte
}

Funding: The author(s) received no specific funding for this work.

Potential competing interests: The author(s) declared that no potential competing interests exist.

\section{Abstract}

Survival of cancer and myeloma patients after diagnosis of medication-related Osteonecrosis of Jaws (ONJ) has not adequately been reported. ONJ is not rare in patients with bone metastatic cancer and myeloma patients, occurring mostly after prolonged treatment with antiresorptive drugs (zoledronic acid and other bisphosphonates, and/or denosumab). On the other hand, expected residual survival can influence the choice of ONJ treatment strategies (surgical versus conservative). We reviewed survival data after ONJ diagnosis time of 609 patients registered in the regional database of a cancer network in North-Western Italy (Rete Oncologica di Piemonte e Valle d'Aosta).

Main characteristics. Disease: metastatic breast cancer 43\%; myeloma 24.1\%; prostate cancer $19.2 \%$; lung cancer 5.7.\% renal cancer 3.2\% other cancer types 4.8\%. Sex: 237 M, 372 F. Median age 69 (32-90) years. Main treatment: $79.1 \%$ zoledronic acid, 4.8\% denosumab; $16.1 \%$ other drugs or drug sequences. Observed actuarial median survival after the registered ONJ diagnosis time was 29 (95\% Cl 26-35) 
months for breast cancer patients, 31 (95\% Cl 28-43) months for myeloma patients, 19 (95\% Cl 15-26) months for prostate cancer patients, 11 (95\% Cl 5-19) months for lung cancer patients, 30 (95\% Cl 1357) months for renal cancer patients, 53 (95\% Cl 17-84) months for other cancer patients.

Two, three and four-year actuarial survival were respectively: 57.6\%, 42.2\%, 33.9\% for breast cancer patients, $64.6 \%, 44.9 \%, 36 \%$ for myeloma patients, $42.7 \%, 27.3 \%, 19.6 \%$ for prostate patients; $28.5 \%$, $14.3 \%, 11.4 \%$ for lung cancer patients; $57.8 \%, 36.8 \%, 26.3 \%$ for renal cancer patients; $62 \%, 58.6 \%$, $58.6 \%$ for others cancer types.

In conclusion, expected survival after ONJ diagnosis is not really short (less than one year) in most of ONJ patients. Our data do not support generalized exclusion of jawbone surgery in ONJ patients due to expected short survival.

Background: There are few literature data about survival of cancer and myeloma patients after diagnosis of Osteonecrosis of Jaws (ONJ) related to antiresorptive treatment (bisphosphonates or denosumab) , with/without biological agents ([1] [2] [3] [4] [5]). Osteonecrosis of Jaws (ONJ) is not rare in patients with bone metastatic cancer and myeloma patients, occurring after prolonged antiresorptive treatment: most of cases are registered after 12-36 months of treatment, even if with a large range (cases observed after few months and other after more than ten years). Furthermore, the expected residual survival after ONJ diagnosis can influence the choice of ONJ treatment strategies: many authors exclude surgery on the base of a supposed short expected survival and for fear of a worsened quality of life and/or impaired ability to tolerate surgery. Some guidelines recommend surgery exclusively for advanced ONJ $\left({ }^{[6]}\right.$, $\left.{ }^{[7]}\right)$, whereas other authors and groups recommend surgery even in early stages of ONJ, to give a better quality of life for patients' residual life time ([8], [9], [10], [11][12]). We analyzed the survival after ONJ diagnosis time in cancer and myeloma patients of a large regional ONJ database ([13], [14])

Materials and Methods: Reports of ONJ patients were retrospectively collected and cross-checked from medical oncology, haematology, and oral care Units of North-Western Italy (Rete Oncologica di Piemonte e Valle d'Aosta), over a 16-year timespan - January 1st, 2003- December 31st, 2018. The following data was collected: M/F ratio, mean age/age range at diagnosis of MRONJ, type of cancer, type of drug - zoledronate, denosumab, others - and date of death, whenever occurred. Kaplan-Meier curves were formulated for survival analysis within each subset of cancer patients, with the aims of acquiring the actuarial median survival, expressed in months, after ONJ diagnosis, as well as the two, three and four-years actuarial survival curves.

Results Data from 609 patients (237 M, 372 F) were acquired. Median age at diagnosis of MRONJ was of 69 (32-90) years, with 127 (20.8\%) under 60 years old, 211 (34.7\%) of 60-70 years old, 271 (44.5\%) over 70 years old.

Underlying diseases were breast cancer (262/609, 43\%), multiple myeloma (147/609, 24.1\%), prostate $(117 / 609,19.1 \%)$ lung cancer $(35 / 609,5.7 \%)$, renal cancer $(19 / 609,3.2 \%)$, other types of cancer $(29 / 609$, 
$4.8 \%)$.

Sex distribution for each cancer was as follows: breast cancer 6 M, 256 F; prostate cancer: 117 M; multiple myeloma: 64 M, 83 F; lung cancer: 18 M, 17 F; renal cancer 15 M 4 F; other cancers: 17 M, 12 F.

Age at ONJ diagnosis for each cancer was as follows: breast cancer $69 \pm 9.1$ (range 32-90) years; prostate cancer $71 \pm 5.9$ (age range 54-86) years; myeloma $72 \pm 7.4$ (range 47-89) years; lung cancer $66 \pm 7.6$ (range: 49-80) years; renal cancer $62 \pm 6.3$ (age range 46-73) years; others $69 \pm 9.6$ (range 40-85) years.

Distribution of main treatment administered was as follows: $79.1 \%$ zoledronic acid, $4.8 \%$ denosumab; 16.1 $\%$ other drugs or drug sequences.

Observed actuarial median survival after ONJ diagnosis was as follows:

- breast cancer 29 (95\% Cl: 26-35) months (Fig 1);

- prostate cancers: 19 (95\% Cl: 15-26) months (Fig 2);

- multiple myeloma 31 (95\% Cl: 28-43) months (Fig 3);

- lung cancer 11 (95\% Cl: 5-19) months;

- renal cancer 30 (95\% Cl: 13-57) months;

- others: 53 (95\% Cl: 17-84) months.

From the actuarial survival curves the following survival rates at 2,3,4 years emerged, respectively: breast cancer : 57.6\%, 42.2\%, 33.9\%; myeloma: $64.6 \%, 44.9 \%, 36 \%$; prostate cancer : 42.7\%, 27.3\%, 19.6\%; lung cancer : $28.5 \%, 14.3 \%, 11.4 \%$; renal

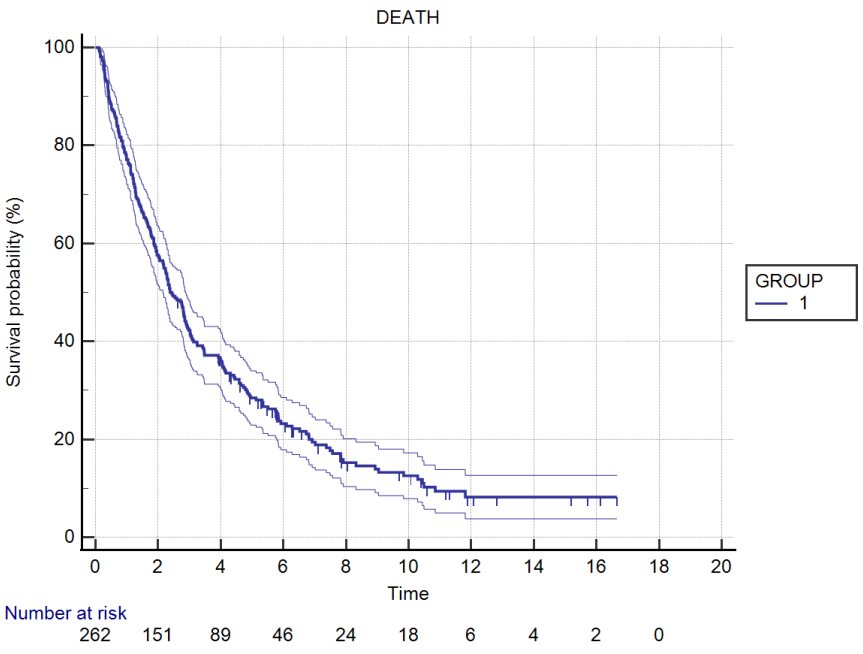

Figure 1 - Breast cancer patients actuarial survival cancer : $57.8 \%, 36.8 \%, 26.3 \%$; other cancers: $62 \%$, $58.6 \%, 58.6 \%$.

Conclusions. : Survival of cancer and myeloma patients after ONJ diagnosis is not short as one could expect on the basis of literature data. Consequently, exclusion of surgical treatment of ONJ disease due to patient expected short survival seems not to be warranted.

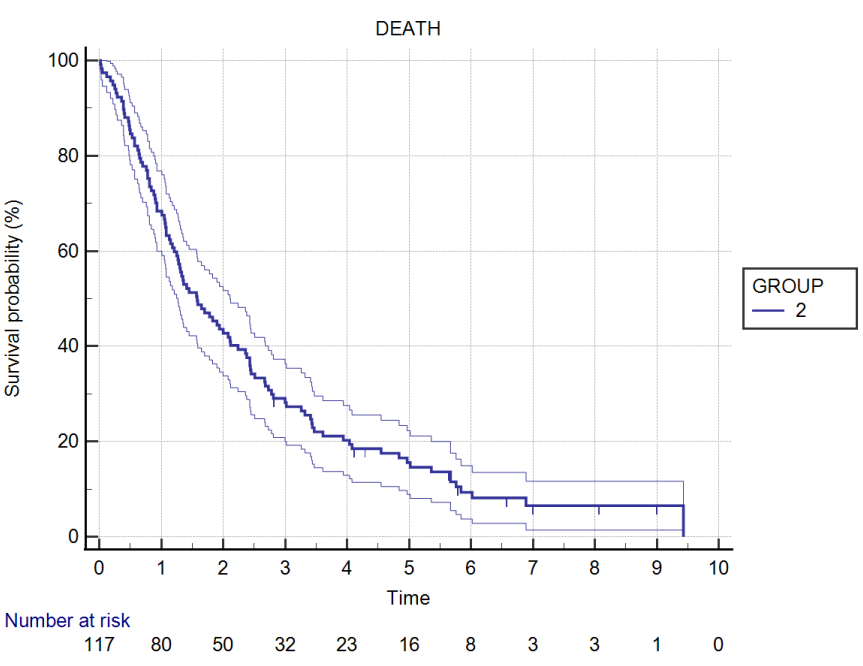

Figure 2 - Prostate cancer patients actuarial survival 


\section{References}

1. `Shin-Yu Lu, Ming-Chun Ma, Ming-Chung Wang, Shui-Sang Hsue. (2021). The status of jaw lesions and medication-related osteonecrosis of jaw in patients with multiple myeloma. Journal of the Formosan Medical Association. doi:10.1016/j.jfma.2021.01.015.

2. ^Ling-Ying Wei, Sang-Heng Kok, Yen-Ching Lee, Wei-Yih Chiu, et al. (2020). Prognosis of medication-related osteonecrosis of the jaws in cancer patients using antiresorptive agent

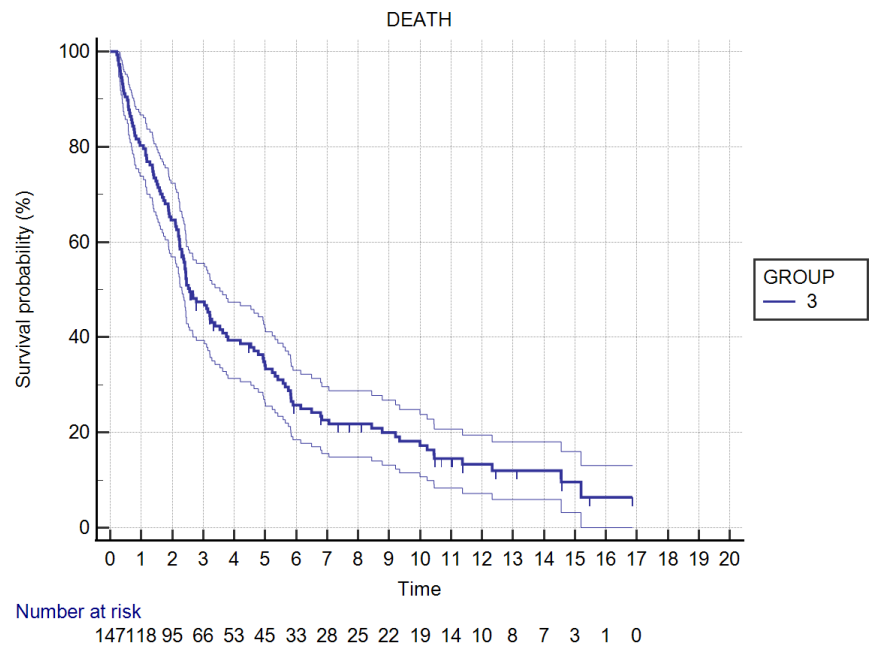
Figure 3 - Myeloma patients actuarial survival zoledronic acid. Journal of the Formosan Medical Association. doi:10.1016/j.jfma.2020.11.017.

3. ^Misato Okamura, Kohei Fujita, Yuki Yamamoto, Osamu Kanai, et al. (2020). Single-center analysis of antiresorptive agent-related osteonecrosis of the jaw in lung cancer patients. Asia-Pac J Clin Oncol, vol. 16 (6), 380-384. doi:10.1111/ajco.13395.

4. `Yasutomo Nakai, Tomohiro Kanaki, Akinaru Yamamoto, Ryo Tanaka, et al. (2020). Antiresorptive agentrelated osteonecrosis of the jaw in prostate cancer patients with bone metastasis treated with bonemodifying agents.J Bone Miner Metab, vol. 39 (2), 295-301. doi:10.1007/s00774-020-01151-9.

5. `Chaoyang Chen, Ruoming Li, Ting Yang, Lingyun Ma, et al. (2020). Denosumab Versus Zoledronic Acid in the Prevention of Skeletal-related Events in Vulnerable Cancer Patients: A Meta-analysis of Randomized, Controlled Trials. Clinical Therapeutics, vol. 42 (8), 1494-1507.e1. doi:10.1016/j.clinthera.2020.05.019.

6. `Salvatore L. Ruggiero, Thomas B. Dodson, John Fantasia, Reginald Goodday, et al. (2014). American Association of Oral and Maxillofacial Surgeons Position Paper on Medication-Related Osteonecrosis of the Law-2014 Update. Journal of Oral and Maxillofacial Surgery, vol. 72 (10), 1938-1956. doi:10.1016/j.joms.2014.04.031.

7. ^Noam Yarom, Charles L. Shapiro, Douglas E. Peterson, Catherine H. Van Poznak, et al. (2019). Medication-Related Osteonecrosis of the Jaw: MASCC/ISOO/ASCO Clinical Practice Guideline.JCO, vol. 37 (25), 2270-2290. doi:10.1200/jco.19.01186.

8. `Alberto Bedogni, Giorgia Saia, Giordana Bettini, Anita Tronchet, et al. (2011). Long-term outcomes of surgical resection of the jaws in cancer patients with bisphosphonate-related osteonecrosis. Oral Oncology, vol. 47 (5), 420-424. doi:10.1016/j.oraloncology.2011.02.024.

9. `Morten Schiodt, Sven Otto, Stefano Fedele, Alberto Bedogni, et al. (2019). Workshop of European task force on medication-related osteonecrosis of the jaw-Current challenges. Oral Dis, vol. 25 (7), 18151821. doi:10.1111/odi.13160. 
10. `Sven Otto, Christoph Pautke, Tim Van den Wyngaert, Daniela Niepel, et al. (2018). Medication-related osteonecrosis of the jaw: Prevention, diagnosis and management in patients with cancer and bone metastases. Cancer Treatment Reviews, vol. 69 , 177-187. doi:10.1016/j.ctrv.2018.06.007.

11. ^Bedogni A, Campisi G, Fusco V, Agrillo A. (2013). Raccomandazioni clinico-terapeutiche sull'osteonecrosi delle ossa mascellari associata a bisfosfonati e sua prevenzione.

12. `Campisi G, Bedogni A, Fusco V. (2020). Raccomandazioni clinico-terapeutiche sull'osteonecrosi delle ossa mascellari (ONJ) farmaco-relata e sua prevenzione.

13. `Vittorio Fusco, Claudia Galassi, Alfredo Berruti, Cinzia Ortega, et al. (2013). Decreasing Frequency of Osteonecrosis of the Jaw in Cancer and Myeloma Patients Treated with Bisphosphonates: The Experience of the Oncology Network of Piedmont and Aosta Valley (North-Western Italy). ISRN Oncology, vol. 2013 , 1-8. doi:10.1155/2013/672027.

14. `V. Fusco, M. Cabras, F. Erovigni, A. Dell'Acqua, et al. (2020). A multicenter observational study on Medication-Related Osteonecrosis of the Jaw (MRONJ) in advanced cancer and myeloma patients of a cancer network in North-Western Italy. Med Oral. doi:10.4317/medoral.24318. 